\title{
Prosthetic Valve Endocarditis after Transcatheter Aortic Valve Implantation Complicated by Paravalvular Abscess and Treated by Pericardial Patches and Sutureless Valve Replacement
}

\author{
Emilien Philippe Ruchonnet, Aurélien Roumy, MD, Valentina Rancati, MD, Matthias Kirsch, MD, PhD \\ Cardiac Surgery Unit, Centre Hospitalier Universitaire Vaudois (CHUV), Lausanne, Switzerland
}

\section{ABSTRACT}

Background: Endocarditis is a rare complication of transcatheter aortic valve implantation (TAVI), with an estimated 1 -year incidence of $0.50 \%$ [Circulation 2015]. However, ensuing consequences are often dire, and its surgical treatment poses unique problems, due to the frequent underlying frailty of TAVI recipients.

Case Report: We report the case of an 84-year-old woman, who developed Staphylococcus aureus prosthetic valve endocarditis (PVE) 7 days after transfemoral TAVI (ACURATE $n e 0^{\mathrm{TM}}$, Boston Scientific or Symetis, further complicated by an aortic annular abscess with fistulization into the right atrium. The patient underwent successful operative aortic annulus repair, using pericardial patches, and aortic valve replacement with a sutureless Perceval S bioprosthesis (LivaNova).

Conclusion: Our case documents the treatment of an active fistulizing Symetis ACURATE $n e 0^{\mathrm{TM}}$ prosthetic aortic valve endocarditis by using a sutureless LivaNova Perceval $\mathrm{S}$ prosthesis with satisfying hemodynamic results and an encouraging 1 -year outcome, further corroborating its usefulness in such circumstances.

\section{INTRODUCTION}

Endocarditis is a rare complication of transcatheter aortic valve implantation (TAVI), with an estimated 1-year incidence of $0.50 \%$ [Amat-Santos 2015]. However, ensuing consequences are often dire, and its surgical treatment poses unique problems, due to the frequent underlying frailty of TAVI recipients. For these patients, a sutureless aortic valve replacement could be an attractive option, because it combines the advantages of expeditious implantation and insertion of a lower amount of prosthetic material. Nevertheless, sutureless aortic valves are contraindicated in patients with endocarditis and aortic annular abscess because of great

Received December 12, 2018; received in revised form February 19, 2019; accepted February 19, 2019.

Correspondence: Mr. Emilien Philippe Ruchonnet Chemin du Grand-Champ 4, 1267 Coinsins, Switzerland; +41-794517189; fax +41-213142278 (e.ruchonnet@bluewin.ch). concern for an insufficient anchoring of the device and subsequent device migration out of a weakened aortic annulus [Gersak 2016].

We report the case of an 84-year-old woman, who developed Staphylococcus aureus prosthetic valve endocarditis (PVE) 7 days after transfemoral TAVI (ACURATE neo ${ }^{\mathrm{TM}}$, Boston Scientific, Marlborough, MA, USA, or Symetis Ecublens, Switzerland [Symetis acquired by Boston Scientific in 2017]), further complicated by an aortic annular abscess with fistulization into the right atrium. The patient underwent successful operative aortic annulus repair, using pericardial patches, and aortic valve replacement with a sutureless Perceval S bioprosthesis (LivaNova, Milan, Italy).

\section{CASE REPORT}

An 84-year-old white woman consulted the emergency department about a New York Heart Association (NYHA) class III dyspnea and intermittent profuse sudation. Her medical history included treated systemic arterial hypertension, and dyslipidemia. Seven days before presentation, she had undergone a transfemoral TAVI using a size M $(25 \mathrm{~mm})$ ACURATE neo ${ }^{\mathrm{TM}}$ valve (Boston Scientific or Symetis), because of severe aortic stenosis with calcified leaflets (with a mean gradient of $24 \mathrm{mmHg}$ and an aortic valve area surface of $0.63 \mathrm{~cm}^{2}$ ) and moderate regurgitation, causing respectively syncopes and NYHA class III dyspnea.

Upon physical examination, the patient was normotensive, apyretic, and otherwise healthy. Laboratory testing revealed an inflammatory syndrome. Transthoracic echocardiography (TTE) showed no signs of endocarditis, with a normal left ventricular ejection fraction (LVEF) of $65 \%$, and a minor aortic paravalvular leak.

Her condition rapidly worsened, with a decrease of her level of consciousness, associated with an acute confusional state, necessitating a transfer to the intensive care unit; the patient underwent a lumbar puncture, the analysis of which suggested bacterial meningitis. Hemocultures were drawn, and an empirical antibiotic treatment of trimethoprimsulfamethoxazole, ceftriaxone, acyclovir, and dexamethasone, was initiated. All (6 of 6 ) hemoculture samples were positive for multisensitive $S$. aureus. Guided by the antibiogram, antibiotic treatment was substituted for one of flucloxacillin, 


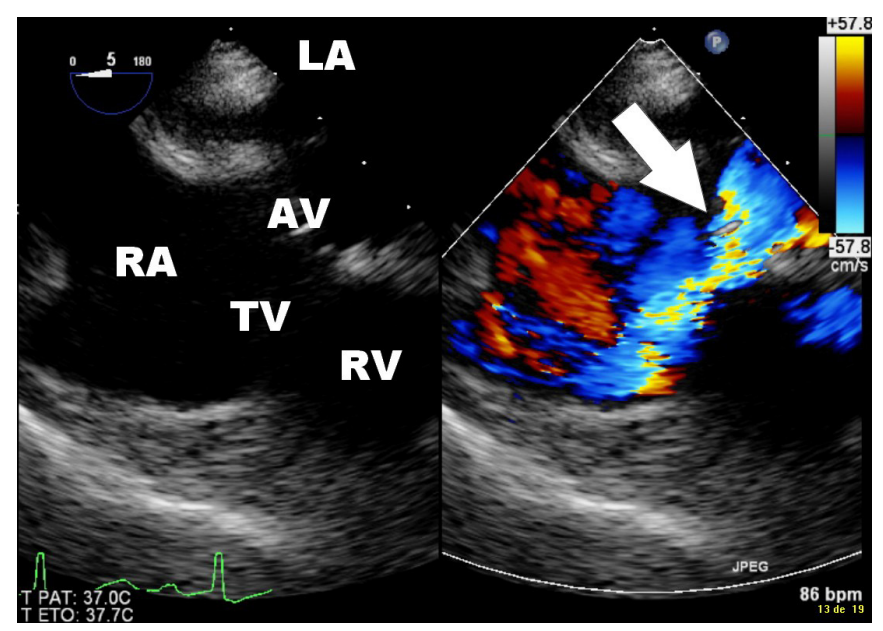

Figure 1. Preoperative midesophageal 5-chamber view showing fistulae between the non- and right-coronary leaflets into the right atrium (immediately above the tricuspid valve). Arrow indicates fistula; AV, aortic valve; LA, left atrium; RA, right atrium; RV, right ventricle; TV, tricuspid valve.

gentamicin, and rifampicin, resulting in negative cultures a day later. However, gentamicin had to be discontinued after 4 days, because of progressive renal failure secondary to the renal toxicity of this agent.

The patient's neurological state rapidly amended. However, a transesophageal echocardiogram (TEE) at post-TAVI day 11 (D11) revealed a worsening paravalvular leak (judged to be moderate) involving $20 \%$ of the valve circumference and a 4-mm thickening of the mitro-aortic junction, suggestive of the development of a paravalvular abscess or of a postprocedural hematoma. Soon, the patient presented an asymptomatic first-degree atrioventricular block and a right bundle branch block. Another TEE at post-TAVI D25 discovered a left-to-right fistula circulating from the left ventricular outflow tract (LVOT) to the right atrium (a Gerbode fistula), which sanctioned surgical correction.

The patient's preoperative EuroSCORE II was 23.6\%. By using median sternotomy, $\mathrm{CPB}$ was established via the ascending aorta and by means of bicaval venous drainage. Cardiac arrest was induced by an intermittent anterograde cold blood cardioplegia. Through the unloaded aortic wall, the distal edge of the TAVI stent was palpated, and a transverse aortotomy was performed $1 \mathrm{~cm}$ distally to it. The Symetis ACURATE $n e 0^{\mathrm{TM}}$ prosthetic valve was extracted, and the native aortic valve leaflets were resected. Upon examination, the prosthesis appeared free of vegetations, but covered with thrombotic material. Culture of the explanted material remained sterile.

Two annular abscesses were identified at the level of the interleaflet triangles: the first, between the non- and left-coronary leaflets; the second, between the non- and right-coronary leaflets and with fistulization into the right atrium, immediately above the tricuspid valve (Figure 1). The aortic annulus was reconstructed by using 2 triangular pericardial patches

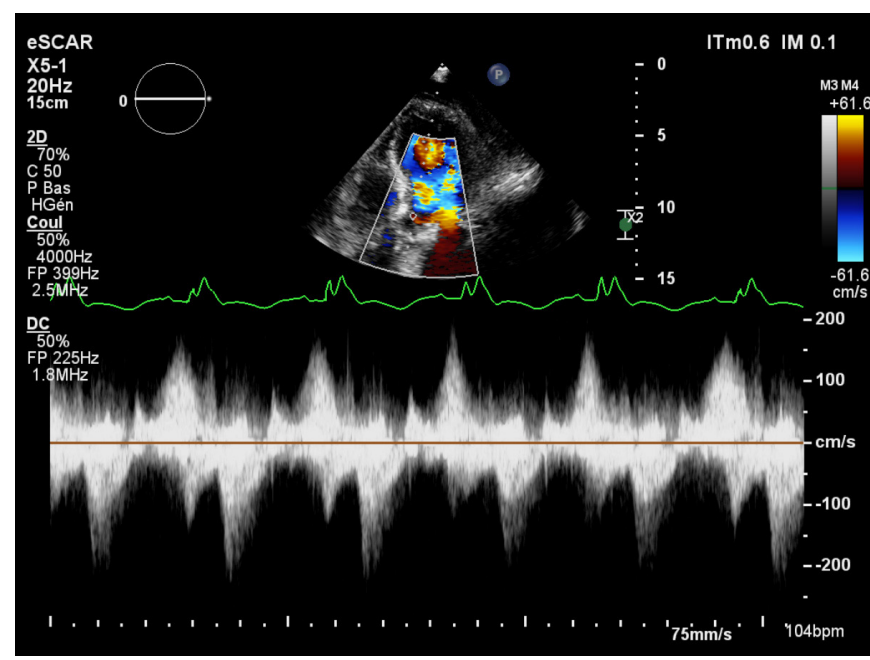

Figure 2. Postoperative transthoracic 5-chamber apical view showing good func-tion of Perceval S sutureless valve.

(XenoSure® Biologic Vascular Patch, made of bovine pericardium, LeMaitre Vascular, Burlington, MA, USA), sutured in place with 5.0 polypropylene running sutures.

The reconstructed aortic root admitted a sutureless LivaNova Perceval S size L (of 24-25 mm) bioprosthesis. The collapsed bioprosthesis was deployed in the LVOT and postdilated by using a dedicated balloon catheter at 4 atm for a duration of 30 seconds. Total $\mathrm{CPB}$ time was 70 minutes; aortic cross-clamp (ACC) time was 59 minutes. Postoperative TEE confirmed the adequate placement of the Perceval $S$ valve, with a mild central regurgitation, and maximal and mean gradients of 8 and $5 \mathrm{mmHg}$, respectively. No residual shunt was present. After 48 hours in our intensive care unit, the patient was transferred after 15 days to a peripheral hospital for convalescence.

During the postoperative period, a complete atrioventricular block required the implantation of a Kora 250 DR pacemaker (Sorin, Milan, Italy). Antibiotics after discharge consisted of flucloxacillin and rifampicin for another 12 days; total duration of antibiotic regimen was 43 days. At discharge, TTE showed maximal and mean gradients of 23 and $11 \mathrm{mmHg}$, respectively, with trivial regurgitation (Figure 2).

At the 1-year follow-up, the patient had no cardiac symptoms and was without signs of recurrent endocarditis. On performance of TTE, LVEF was estimated at $55 \%$, and the function of the prosthetic valve was defectless (with maximal and mean gradients of 10 and $5 \mathrm{mmHg}$, respectively) and had no leakage.

\section{DISCUSSION}

Treatment of PVE is an arduous process. Antibiotic therapy alone often fails to eradicate the infection, because it is entrenched in foreign material, and complications (such as abscesses, fistulization, or valve function deterioration) 
Use of Perceval S Sutureless Valve in Patients with Aortic Annular Abscess(es)*

\begin{tabular}{lcccccc}
\hline Authors (Year) & Number & NVE & PVE & Annular Abscess & Patch Repair & VAl $(\geq 2 / 4)$ \\
\hline Lio et al (2015) & 5 & 0 & 5 & 3 & 1 & 0 \\
Weymann et al (2017) & 9 & 5 & 4 & 4 & 4 & 0 \\
Roselló-Díez et al (2018) & 9 & 0 & 9 & 7 & 1 & 2 \\
\hline
\end{tabular}

*NVE, native valve endocarditis; PVE, prosthetic valve endocarditis; VAl, intra- or paravalvu-lar aortic insufficiency; IHD, in-hospital death(s).

frequently occur. Thus, surgical correction of PVE, with the removal of the infected prosthesis and its subsequent replacement, becomes a key component in infection control.

Management of staphylococcal TAVI PVE, according to the European Society of Cardiology 2015 guidelines [Habib 2015], should consist of a regimen of at least 6 weeks of flucloxacillin, rifampicin, and gentamicin.

Although TAVI PVE management guidelines are identical to those for other PVE instances, such cases present with a specific difficulty: patients who benefited from a TAVI were, by definition, ineligible for surgical replacement. Thus, emergency surgery performed in these circumstances on infected tissue often yields a poor prognosis. Luckily, PVE remains a rare complication of TAVI, with an estimated 1-year incidence of $0.50 \%$ [Amat-Santos 2015].

One potential reason for the lower incidence of PVE after TAVI in comparison to surgical aortic valve replacement (SAVR) is that a TAVI requires a relatively low amount of implanted prosthetic material. Less prosthetic material can be expected to reduce the risk of infection. The ACURATE $n e 0^{\mathrm{TM}}$ valve is a second-generation TAVI device designed to lessen the risk of paravalvular leakage reported with earlier models [Husser 2017]. For that purpose, it is fitted with significantly larger pericardial skirts. This additional amount of bioprosthetic tissue might increase the risk of infection, and future studies should compare infection rates between first-generation and more recent TAVI devices to assess the accuracy of this hypothesis.

Replacement of an infected prosthetic valve with conventional prostheses has proven to be adequate, with an early mortality rate of $9.7 \%$, and satisfying 5 - and 10 -year survival rates [Perrotta 2016]. Principles of surgical treatment of PVE include meticulous debridement of all infected tissues, and removal of all foreign material. In some patients, this leaves an extremely fragile aortic annulus, through which suture placement can be arduous. In these cases, numerous stitches-eventually reinforced with felt pledgets or pericardium - have to be used. This allows possible postoperative suture dehiscence and a higher risk of recurrent infection. For these reasons, the use of a sutureless valve, combining the advantage of an expeditious implantation with the absence of additional prosthetic material (as with a TAVI device), could be justified. However, a weakened aortic annulus might compromise adequate anchoring of the device, which relies solely on radial force, and exposes the patient to paravalvular leak and/or device migration. For these reasons, infective endocarditis complicated by annular abscess has been considered an absolute contraindication to sutureless valve or rapid deployment valve placement [Gersak 2016]. Our case shows that the Perceval S sutureless valve can be used safely, even in a patient with multiple aortic annular abscesses requiring pericardial patch repair. Other groups have reported their experience in this indication [Lio 2015; Weymann 2017; Roselló-Díez 2018], summarized in the Table.

Overall, the published results are encouraging, with a low rate of need of device redeployment or paravalvular leak and with no reports of late device migration or recurrent infection. More studies are needed to ascertain that such an approach, using a sutureless valve (a relatively recent technique), truly represents an adequate alternative to other existing methods using former prosthetis models. Moreover, long-term followup of these patients is necessary, because late paravalvular leak prosthesis dysfunction may occur.

In conclusion, Perceval S sutureless valves appear to be a safe alternative to conventional bioprostheses in the treatment of active PVE, even when complicated by an annular abscess. It may allow shortening of ACC and CPB times, which might be of a particular advantage in frail patients undergoing complex procedures in a critical state.

\section{VIDEO CLIP OF PERCEVAL S SUTURELESS VALVE IN PLACE}

Video clip showing a color Doppler short-axis view of the Perceval S sutureless valve in place (see https://vimeo. com/328439706.

\section{REFERENCES}

Amat-Santos IJ, Messika-Zeitoun D, Eltchaninoff H, et al. 2015. Infective endocarditis after transcatheter aortic valve implantation: results from a large multicenter registry. Circulation 131(18):1566-74.

Gersak B, Fischlein T, Folliguet TA, et al. 2016. Sutureless, rapid deployment valves and stented bioprosthesis in aortic valve replacement: recommendations of an International Expert Consensus Panel. Eur J Cardiothorac Surg 49(3):709-18.

Habib G, Lancellotti P, Antunes MJ, et al. 2015.2015 ESC Guidelines for the management of infective endocarditis: the Task Force for the Management of Infective Endocarditis of the European Society of Cardiology (ESC). Endorsed by: European Association for Cardio-Thoracic Surgery 
(EACTS), the European Association of Nuclear Medicine (EANM). Eur Heart J 36(44):3075-128.

Husser O, Kim WK, Pellegrini C, et al. 2017. Multicenter comparison of novel self-expanding versus balloon-expandable transcatheter heart valves. JACC Cardiovasc Interv 10(20):2078-87.

Lio A, Miceli A, Solinas M, Glauber M. 2015. Initial experience with sutureless Sorin Perceval S aortic prosthesis for the treatment of prosthetic valve endocarditis. Thorac Cardiovasc Surg 63(6):501-3.

Perrotta S, Jeppsson A, Fröjd V, Svensson G. 2016. Surgical treatment of aortic prosthetic valve endocarditis: a 20-year single-center experience. Ann Thorac Surg 101(4):1426-32.

Roselló-Díez E, Cuerpo G, Estévez F, et al. 2018. Use of the Perceval sutureless valve in active prosthetic aortic valve endocarditis. Ann Thorac Surg 105(4):1168-74.

Weymann A, Konertz J, Laule M, Stangl K, Dohmen PM. 2017. Are sutureless aortic valves suitable for severe high-risk patients suffering from active infective aortic valve endocarditis? Med Sci Monit 23:2782-7. 